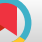

\title{
Gene Expression Analysis of Key Players Associated with Fluconazole Resistance in Candida albicans
}

\author{
Hongxia Zhang ${ }^{1}$, Qiufang $\mathrm{Xu}^{2}$, Suchen $\mathrm{Li}^{1}$, Ying Ying ${ }^{1}$, Zhiqin Zhang ${ }^{3}$, Lingbing Zeng ${ }^{4}$, Xiao-Tian \\ Huang ${ }^{1}$ and Shibo Huang ${ }^{1,5,{ }^{*}}$ \\ ${ }^{1}$ Jiangxi Medical College, Nanchang University, Nanchang, China \\ ${ }^{2}$ Qingpu District Center for Disease Control and Prevention, Shanghai, China \\ ${ }^{3}$ Pingxiang Second People's Hospital, Pingxiang, China \\ ${ }^{4}$ Department of Clinical Laboratory, The First Affiliated Hospital of Nanchang University, Nanchang University, Nanchang, China \\ ${ }_{5}^{5}$ Jiangxi Province Key Laboratory of Tumor Pathogens and Molecular Pathology and Department of Pathology, Schools of Basic Medical Sciences and Pharmaceutical \\ Sciences, Nanchang University Medical College, Nanchang, China \\ "Corresponding author: Jiangxi Medical College, Nanchang University, 461 Bayi Road, Nanchang 330006, China. Tel: +86-79186360654, Email: hsb@ncu.edu.cn
}

Received 2018 December 31; Revised 2019 July 24; Accepted 2019 July 28.

\begin{abstract}
Background: Fluconazole resistance in Candida albicans has become a serious public health problem. Most previous studies have focused on deciphering the relationship between fluconazole resistance and amino acid substitutions in ERG11, which encodes cytochrome P450 lanosterol $14 \alpha$-demethylase whose enzymatic activity is inhibited by azoles. However, azole resistance in C. albicans is a multifactorial phenomenon and several lines of evidence indicate that other genes and mechanisms may contribute to the development of fluconazole resistance.

Objectives: The present study aimed to investigate the underlying role of six genes in fluconazole-resistant clinical strains of $C$. albicans, including ERG11, RTA2, and the efflux pump genes CDR1, CDR2, MDR1, and FLU1.

Methods: We collected 40 fluconazole-resistant isolates and 40 susceptible isolates from patients with Candida infections in the First Affiliated Hospital of Nanchang University in China from 2005 to 2008. The susceptibility of the isolates to antifungal agents was tested by the M27-A3 broth microdilution method following the Clinical and Laboratory Standards Institute (CLSI) guidelines. Then, the gene expression levels of several key players in azole resistance were quantified.

Results: Most fluconazole-resistant strains analyzed in this study were found to be cross-resistant to ketoconazole, itraconazole, and clotrimazole. We observed that the FLU1 gene expression significantly increased $(\mathrm{P}<0.05)$ and exhibited major changes in most of the fluconazole-resistant isolates (75.0\%). In addition, the expression of a novel gene, RTA2, was remarkably upregulated (P $<0.05$ ). Interestingly, we found that $10 \%$ of the fluconazole-resistant isolates were simultaneously associated with ERG11 mutation and overexpression of RTA2, CDR1, and FLU1 genes. Unlike other studies, we did not find any difference in the expression of CDR2, $M D R 1$, and ERG11 genes between the fluconazole-susceptible and resistant isolates.

Conclusions: Our findings suggested that the overexpression of FLU1 and RTA2 genes may cause azole resistance; this finding had not been reported previously in clinical isolates of C. albicans. The upregulation of FLU1 and RTA2 genes was the predominant mechanism of fluconazole resistance in C. albicans in China.
\end{abstract}

Keywords: Candida albicans, Fluconazole, Drug resistance, ATP-Binding Cassette Transporter

\section{Background}

Candida albicans is a predominant fungal opportunistic pathogen responsible for both mucosal and systemic infections, and is especially prevalent in immunodeficient hosts (1-4). Infections caused by C. albicans are mainly treated with azoles, which include both imidazoles (miconazole, clotrimazole, and ketoconazole) and triazoles (fluconazole and itraconazole). However, the long-term, repeated usage of azoles has resulted in the emergence of resistant isolates $(5,6)$. Drug resistance is a serious complication that can be challenging to clinicians, posing a major hurdle in the success of antifungal therapy (7). Previous studies have shown that decreased susceptibility to azoles is mainly associated with alterations in the ERG11 gene and/or constitutive upregulation of multidrug efflux pumps $(8,9)$. Azoles act as non-competitive ERG11 inhibitors through coordination with the iron atom of the heme group located in the active site of $14-\alpha$-sterol demethylase.

Numerous publications have shown that non- 
synonymous point mutations in ERG11 may lead to conformational changes, resulting in the decreased affinity of azoles for the 14- $\alpha$-sterol demethylase enzyme (9-11). In addition, elevated gene expression levels of efflux transporters can reduce the intracellular accumulation of certain drugs, and is often the main mechanism of azole resistance in clinical Candida (12). There are two families of membrane-associated efflux pumps, including ATP-binding cassette (ABC) superfamily (12) and major facilitator superfamily (MFS) (13), which have been found to be upregulated in resistant isolates. Two major $A B C$ transporter genes, CDR1 (14) and CDR2 (Candida Drug Resistance1 and 2), are well-documented in clinical drug resistance. Both genes have shown to be upregulated in azoles-resistant $C$. albicans isolates and the genetic deletion of both genes in C. albicans results in hypersensitivity to azoles (15-19).

The expression levels of MDR1 (Multidrug resistance1) and FLU1 (Fluconazole resistance1), which are the members of the MFS, have also shown to be specifically upregulated in azole-resistant $C$. albicans strains $(20,21)$. In addition, several other factors can contribute to C. albicans azole resistance, including processes involved in biofilm formation and mutations in other enzymes in the ergosterol pathway (22). More recently, a novel gene (resistant to 7-aminocholesterol, RTA2), which has also shown to contribute to the development of azole resistance, has been identified in a mutant strain lacking the CDR1, CDR2, and MDR1 genes (23). Taken together, it is apparent that drug resistance is a multifactorial phenomenon. Due to the increased number of resistant strains, it is increasingly important to investigate the underlying mechanisms of azole resistance for the development of new antifungal treatments for C. albicans.

\section{Objectives}

In our previous study, we sequenced several fluconazole-resistant clinical isolates and found that 63.27\% of the isolates did not contain mutated ERG11 (24). Our findings strongly suggested that other resistance mechanisms exist that require further research. In this study, we first analyzed the susceptibility of these isolates to other antifungal agents, and then investigated the relationship between fluconazole resistance, alternations in the ERG11 gene, and the expression levels of several membrane-associated efflux pumps (CDR1, CDR2, MDR1, FLU1) and RTA2 genes to decipher the potential molecular mechanisms leading to fluconazole-resistance in these clinical C. albicans strains.

\section{Methods}

\subsection{Strains}

We selected 40 fluconazole-resistant $C$. albicans isolates from our previous study using standard drug susceptibility analysis assays, defined herein as the fluconazoleresistant group. Additionally, 40 C. albicans isolates, which were susceptible to all drugs evaluated in our study, were defined as the susceptible group. All the C. albicans isolates were obtained from patients undergoing treatment for respiratory, genital, bloodstream, urinary, and digestive tract infections from 2005 to 2008 at the First Affiliated Hospital of Nanchang University in China. For drug susceptibility analysis, we used C. krusei ATCC 6258 and C. parapsilosis ATCC 22019 as quality control reference strains. Fluconazole-sensitive C. albicans ATCC 90028 was included as a control for the analysis of gene expression.

\subsection{Antifungal Agents}

Ketoconazole, itraconazole, 5-flucytosine, amphotericin B, clotrimazole, and nystatin were obtained from Sigma-Aldrich (USA). The measurable concentrations of ketoconazole, itraconazole, amphotericin B, clotrimazole, and nystatin ranged from 0.0313 to $16 \mu \mathrm{g} / \mathrm{mL}$ and dilutions of 5-flucytosine used herein ranged from 0.125 to 64 $\mu \mathrm{g} / \mathrm{mL}$.

\subsection{Drug Susceptibility Analysis}

The antifungal agent susceptibility analysis was performed according to the CLSI Broth Microdilution Susceptibility Method (M 27-A3 Document) for antifungal susceptibility of yeast. Briefly, stock inoculum suspensions of C. albicans were prepared and diluted with RPMI 1640 broth to obtain two inoculums $\left(1 \times 10^{3}\right.$ to $\left.5 \times 10^{3} \mathrm{CFU} / \mathrm{mL}\right)$. Next, we plated $100 \mu \mathrm{L}$ of each suspension into wells already containing $100 \mu \mathrm{L}$ of the given antifungal agent (final inoculum size of $5 \times 10^{2}$ to $2.5 \times 10^{3} \mathrm{CFU} / \mathrm{mL}$ ). Then, the plates were cultured at $35^{\circ} \mathrm{C}$ for $48 \mathrm{~h}$. The resistance breakpoint categories of 5-flucytosine, itraconazole, and fluconazole were used according to the CLSI M27-A3 criteria (25) while ketoconazole and clotrimazole were based on previous investigations (26), as shown in Table 1 . The minimal inhibitory concentrations (MICs) were determined as the lowest concentration causing at least $80 \%$ growth inhibition compared to control wells not containing the antifungal agent. The MICs for amphotericin B and nystatin were identified as the minimum concentration yielding a complete growth inhibition. 
Table 1. The Interpretive Breakpoints of Drug Susceptibility to Fluconazole, 5- Flucytosine, Itraconazole, Ketoconazole, and Clotrimazole

\begin{tabular}{lccc}
\hline Drugs & $\mathbf{S}, \mu \mathbf{g} / \mathbf{m L}$ & $\mathbf{S}-\mathbf{D D}, \mu \mathbf{g} / \mathbf{m L}$ & $\mathbf{R}, \mu \mathbf{g} / \mathbf{m L}$ \\
\hline Fluconazole (25) & $\leq 8$ & $16 \sim 32$ & $\geq 64$ \\
\hline 5-flucytosine (25) & $\leq 4$ & $8 \sim 16$ & $\geq 32$ \\
\hline Itraconazole (25) & $\leq 0.125$ & $0.25 \sim 0.50$ & $\geq 1.0$ \\
Ketoconazole (26) & $\leq 0.125$ & $0.25 \sim 0.50$ & $\geq 1.0$ \\
Clotrimazole (26) & & & $\geq 0.5$ \\
\hline
\end{tabular}

\subsection{RNA Extraction}

Total cellular RNA was isolated from C. albicans at midexponential growth phase $\left(\mathrm{OD}_{600}=0.8\right)$ using the hot phenol method (27). Briefly, cells were collected by centrifugation at $4000 \mathrm{rpm}$ for $5 \mathrm{~min}$ at room temperature, the supernatant was removed, and then the pellets were spun down a second time to remove all liquid. Next, cell pellets were resuspended in $800 \mu \mathrm{L}$ of AE buffer ( $50 \mathrm{~mm}$ sodium acetate, $10 \mathrm{~mm}$ EDTA, pH 5.2). Then, $80 \mu \mathrm{L}$ of $10 \%$ SDS and $880 \mu \mathrm{L}$ of pre-warmed phenol ( $\mathrm{pH}$ 5.2) were added and the samples were vortexed thoroughly. The tubes were transferred to a $65^{\circ} \mathrm{C}$ water bath and vortexed for $5 \mathrm{~s}$ once every $1 \mathrm{~min}$ for $5 \mathrm{~min}$. Lysates were transferred and kept in a dry ice/ethanol bath for 2-3 min, followed by centrifugation at $13,200 \mathrm{rpm}$ for $10 \mathrm{~min}$ at room temperature.

The aqueous layers were carefully transferred to new microcentrifuge tubes and an equal volume of phenol ( $\mathrm{pH}$ 5.2): chloroform: isoamyl alcohol (25: 24: 1) was added. Then, samples were mixed by vortexing and centrifuged again at 13,200 rpm for $10 \mathrm{~min}$ at room temperature. The aqueous layers were then carefully transferred to new microcentrifuge tubes. RNA was precipitated by the addition of 1/10 volume of $3 \mathrm{M}$ sodium acetate ( $\mathrm{pH} 5.2$ ) and 2.5 volume of chilled $100 \%$ ethanol, followed by incubation at $20^{\circ} \mathrm{C}$ overnight. Precipitated RNA was collected by centrifugation at 13,200 rpm for $15 \mathrm{~min}$ at $4^{\circ} \mathrm{C}$. The entire supernatant was decanted; the RNA pellet was washed with 1 $\mathrm{mL}$ of chilled $75 \%$ ethanol and centrifuged again. The pellet containing RNA was air-dried in a hood for 5 - 10 min and then resuspended in $100 \mu \mathrm{L}$ of DEPC water; then, it was treated with RNase-free DNase (ThermoFisher, USA) to prevent genomic DNA contamination. The quantity and quality of RNA were assessed by the measurement of the OD 260/280 absorption ratio and running the denaturing formaldehyde agarose gel.

\subsection{Quantitative Real-Time PCR}

The cDNA was synthesized using $2 \mu \mathrm{g}$ of total RNA according to the manufacturer's instruction of the PrimeScript ${ }^{\mathrm{TM}}$ RT Reagent kit with gDNA Eraser (Takara, Japan). Gene-specific PCR primers for RTA2, CDR1, CDR2,
ERG11, FLU1, and MDR1 genes and 18S rRNA were designed using Primer Premier 5.0 (Table 2). The 18S rRNA was included as an internal reference gene. The qRT-PCR analysis was performed using the 7500 Real-time PCR System (Applied Biosystems, USA) and the SYBR ${ }^{\circledR}$ Premix Ex Taq $^{\mathrm{TM}}$ II (Tli RNaseH Plus) kit (Takara, Japan). All samples contained $10 \mu \mathrm{L}$ of $2 X$ SYBR Premix Ex Taq ${ }^{\mathrm{TM}}$ II (Tli RNaseH Plus) (including TaKaRa Ex Taq ${ }^{\circledR} \mathrm{HS}$, dNTP Mixture, $\mathrm{Mg}^{2+}$, Tli RNaseH, SYBR ${ }^{\circledR}$ Green I), $0.8 \mu \mathrm{L}$ of forward and reverse primers, $1 \mu \mathrm{L}$ of cDNA template, $0.4 \mu \mathrm{L}$ of ROX Reference Dye, and $7.2 \mu \mathrm{L}$ of nuclease-free water.

\begin{tabular}{|c|c|c|}
\hline No. & Primer Name & Sequence (5' - 3') \\
\hline \multirow[t]{2}{*}{1} & CaERG11a-F & CAAGAAGATCATAACTCAAT \\
\hline & CaERG11a-R & CAGAACACTGAATCGAAAGA \\
\hline \multirow[t]{2}{*}{2} & CaERG11b-F & TTTGGTGGTGGTAGACATAGAT \\
\hline & CaERG11b-R & TAATCAGGGTCAGGCACTTT \\
\hline \multirow[t]{2}{*}{3} & CaCDR1-F & GATTCTCAAACTGCCTGGTC \\
\hline & CaCDR1-R & CCAAAATAAGCCGTTCTTCCAC \\
\hline \multirow[t]{2}{*}{4} & CaCDR2-F & AAAAAGGTGGAAGAACGGC \\
\hline & CaCDR2-R & TTGGCATGAGATCCTGGTG \\
\hline \multirow[t]{2}{*}{5} & CaMDR1-F & TGCGTCAAGAACAGGTTITC \\
\hline & CaMDR1-R & AAGCAGTAGTAGCAGCACC \\
\hline \multirow[t]{2}{*}{6} & CaFLU1-F & TGGATAGTCCCCGTCATTGG \\
\hline & CaFLU1-R & GGCAAAAAGTGGGAAAACAGC \\
\hline \multirow[t]{2}{*}{7} & CaRTA2F & ATGTCAAATCGGTAAGAGGTC \\
\hline & CaRTA2R & AGCCAATTCTGCCACTCTAT \\
\hline \multirow[t]{2}{*}{8} & 18S RNA-F & TCTTTCTTGATTTTGTGGGTGG \\
\hline & 18S RNA-R & TCGATAGTCCCTCTAAGAAGTG \\
\hline
\end{tabular}

The PCR conditions were as follows: 40 cycles of denaturation for $5 \mathrm{~S}$ at $95^{\circ} \mathrm{C}$, annealing for $34 \mathrm{~s}$ at $60^{\circ} \mathrm{C}$ for $18 \mathrm{~S}$ rRNA, MDR1, CDR2, CDR1, FLU1, RTA2, and ERG11, and a melt curve step (from $60^{\circ} \mathrm{C}$, gradually increasing at $0.5^{\circ} \mathrm{C} / \mathrm{s}$ to $95^{\circ} \mathrm{C}$, with acquisition data every $1 \mathrm{~s}$ ). Fluorescent data were collected during the annealing step and analyzed with $\mathrm{ABI}$ software. The threshold cycle $(\Delta \mathrm{CT})$ value was obtained by calculating the difference between the CT values of the target gene and the normalizer (18S rRNA). The mean mRNA levels for each gene were calculated from at least three independent biological replicates. The expression levels of target genes were analyzed according to the $2^{-\Delta \Delta \mathrm{Ct}}$ method (28). Differences between resistant and susceptible groups were analyzed with independent sample t-test. 


\subsection{Statistical Analysis}

The results are expressed as means \pm standard deviation(SD). Comparisons between the sensitive and resistant groups were made using independent-samples $t$-tests by SPSS 13.0 software. The differences were considered statistically significant when the P values were less than 0.05 .

\section{Results}

\subsection{Antifungal Susceptibility}

As described in Table 3, all fluconazole-resistant strains were susceptible to 5-flucytosine. Moreover, 25 (67.74\%) fluconazole-resistant isolates were also resistant to ketoconazole, 28 (70.97\%) were resistant to itraconazole, and 23 (64.52\%) were resistant to clotrimazole. Interestingly, the fluconazole-resistant isolates, which were associated with the mutation of ERG11, exhibited a higher MIC for nystatin, while the isolates that were not associated with the mutation of ERG11 were cross-resistant to other azoles.

\subsection{Expression of ERG11}

The melting peaks and curves of the ERG11 amplicon indicated that there were no non-specific amplification products or primer dimers produced during the reactions. Further, a single band representing the PCR product with the expected length on an agarose gel confirmed the specificity of the PCR (data not shown). Gene expression levels of ERG11 from 40 fluconazole-resistant and -susceptible isolates were analyzed by the real-time PCR. The mean $\Delta \mathrm{Ct}$ values of the ERG11 gene in the fluconazole-resistant group and the susceptible group were $16.80 \pm 0.18$ and $17.16 \pm$ 0.26 , respectively, with no statistically significant difference $(P=0.25)$.

\subsection{The Expression of Efflux Pumps}

The CDR1 expression level was 3.68 folds higher in the fluconazole-resistant group than in the fluconazolesusceptible group (Figure 1). The mean $\Delta \mathrm{Ct}$ values of CDR1 expression were $15.92 \pm 0.28$ and $16.80 \pm 0.24$ in the resistant and susceptible groups, respectively. The expression of CDR1 was significantly different between the fluconazole-susceptible and resistant groups $(\mathrm{P}=0.0193)$. Among the 40 fluconazole-resistant isolates, isolate 59679 displayed the highest level of CDR1 gene expression (23.07 folds) (Figure 1B). In our previous work, we found that only $36.73 \%$ (19/49) of the fluconazole-resistant isolates were associated with the mutation in the ERG11 gene. In this study, we further analyzed the differences between isolates related and unrelated to the mutation in the ERG11 gene and found that CDR1 expression was altered in $80.00 \%$ of the fluconazole-resistant isolates containing a mutant variant of ERG11, and that the expression level of CDR1 was more than 1.5-fold higher in these isolates (Figure 1A).

Among the 40 fluconazole-resistant isolates, 31(77.50\%) isolates exhibited 1.29 to 10.50 -fold upregulation of the FLU1 gene (Figure 2). The mean $\triangle \mathrm{Ct}$ values of the FLU1 gene were $17.64 \pm 0.16$ and $18.39 \pm 0.15$, respectively, in the resistant and susceptible groups. The difference in FLU1 gene expression was 0.0010 between fluconazole-resistant and susceptible groups, which was statistically significant ( $\mathrm{P}$ $=0.0010)$. Compared to the susceptible isolates, the expression levels of FLU1 were not upregulated in most resistant isolates, which carried a mutant variant of ERG11. The mean $\triangle \mathrm{Ct}$ values of the CDR2 and MDR1 genes were 15.96 \pm 0.25 and $21.51 \pm 0.31$, respectively, in the resistant group and $16.27 \pm 0.45$ and $21.72 \pm 0.17$, respectively, in the susceptible group. The mRNA expression levels of CDR2 and MDR1 were not statistically different between fluconazoleresistant and susceptible groups $(\mathrm{P}=0.55$ and 0.56 , respectively).

\subsection{Expression of RTA2}

Among 40 fluconazole-resistant isolates, 19 (47.5\%) isolates exhibited more than 1.5 -fold upregulation in the RTA2 gene when compared to the fluconazole susceptible isolates, and the mean RTA2 gene expression in resistant isolates was 4.91 folds higher (Figure 3$)$. The mean $\Delta \mathrm{Ct}$ values of the RTA2 gene were $16.08(\mathrm{SD}=0.28)$ and $17.19(\mathrm{SD}=0.20)$, respectively, in the resistant and susceptible groups, which indicated that RTA2 gene expression was significantly different between these two groups $(\mathrm{P}=0.0017)$.

\section{Discussion}

Azole is a widely used antifungal agent for the treatment of both superficial mucosal and deep disseminated Candida infections. However, the widespread application of azoles and the structural similarity of these molecules have resulted in the development of cross-resistance to various members of this class of drugs (29). Previous studies demonstrated that fluconazole resistance was correlated with cross-resistant to other azoles (30). Recently, researchers found that about $74 \%$ of fluconazole-resistant strains were also resistant to ketoconazole and itraconazole (31). Our present study demonstrated that most fluconazole-resistant isolates were cross-resistant to other azoles.

Resistance to a variety of drugs is defined as multidrug resistance (MDR). In infectious bacteria, such as Mycobacterium tuberculosis, the emergence of MDR is caused by a series of point mutations in different target genes (32). A similar process can be found in fungal infections, as well. 
Zhang Het al.

\begin{tabular}{|c|c|c|c|c|c|c|c|c|}
\hline \multirow{2}{*}{ Isolate } & \multirow{2}{*}{ FlZ } & \multicolumn{6}{|c|}{ MIC, $\mu \mathbf{g} / \mathbf{m L}$} & \multirow{2}{*}{ Amino Acid Change (s) in Erg11p } \\
\hline & & KETO & ITR & CLOT & 5-FC & NYS & $\mathbf{B}$ & \\
\hline 56388 & 64 & 8 & 4 & 2 & 2 & 16 & 4 & A114S Y257H \\
\hline 49372 & 64 & 0.5 & 16 & 0.5 & 2 & 8 & 1 & A114S Y257H \\
\hline 49922 & 64 & 0.5 & 4 & 0.25 & 0.125 & 8 & 1 & A114S Y257H \\
\hline 56539 & 64 & 0.25 & 0.5 & 0.25 & 1 & 8 & 1 & A114S Y257H \\
\hline 57451 & 64 & 1 & 2 & 4 & 0.25 & 4 & 4 & A114S Y257H \\
\hline 57800 & 64 & 0.0625 & 0.5 & 0.625 & 0.125 & 4 & 2 & A114S Y257H \\
\hline 58181 & 64 & 1 & 4 & 0.25 & 0.125 & 4 & 2 & A114S Y257H \\
\hline 59161 & 64 & 0.25 & 2 & 0.25 & 0.125 & 4 & 2 & A114S Y257H \\
\hline 59182 & 64 & 0.5 & 1 & 2 & 4 & 8 & 1 & A114S Y257H \\
\hline 56472 & 64 & 16 & 2 & 0.25 & 0.25 & 0.125 & 0.5 & D116E K128T Y132H G465S \\
\hline 59690 & 64 & 16 & 8 & 0.25 & 0.5 & 8 & 2 & D116E K128T Y132H G465S \\
\hline 51527 & 64 & 1 & 0.5 & 0.25 & 4 & 2 & 2 & G450E Y132H \\
\hline 58614 & 64 & 0.0313 & 0.0313 & 0.25 & 0.125 & 16 & 4 & Y132H G450E \\
\hline 56392 & 64 & 2 & 16 & 0.5 & 0.125 & 8 & 4 & Y132H G488E \\
\hline 56292 & 64 & 8 & 16 & 1 & 0.125 & 4 & 1 & A114S Y257H \\
\hline 54535 & 64 & 0.5 & 0.125 & 0.125 & 0.125 & 2 & 2 & $\mathrm{a}^{\mathrm{a}}$ \\
\hline 49340 & 64 & 8 & 4 & 2 & 1 & 2 & 1 & $\mathrm{a}^{\mathrm{a}}$ \\
\hline 49345 & 64 & 0.5 & 16 & 0.5 & 0.25 & 4 & 1 & $\mathrm{a}^{\mathrm{a}}$ \\
\hline 56350 & 64 & 16 & 16 & 2 & 0.125 & 1 & 4 & $\mathrm{a}^{\mathrm{a}}$ \\
\hline 56525 & 64 & 8 & 16 & 1 & 0.125 & 4 & 4 & $\mathrm{a}^{\mathrm{a}}$ \\
\hline 57598 & 64 & 8 & 16 & 0.5 & 0.25 & 4 & 4 & $\mathrm{a}^{\mathrm{a}}$ \\
\hline 49312 & 64 & 16 & 0.25 & 0.25 & 0.125 & 2 & 1 & D116E \\
\hline 49477 & 64 & 0.5 & 16 & 0.25 & 0.125 & 4 & 1 & D116E \\
\hline 56452 & 64 & 16 & 4 & 0.0625 & 0.25 & 0.125 & 2 & D116E \\
\hline 56477 & 64 & 16 & 16 & 0.25 & 0.125 & 4 & 4 & D116E \\
\hline 56507 & 64 & 8 & 16 & 0.5 & 0.5 & 4 & 4 & D116E \\
\hline 56533 & 64 & 16 & 16 & 0.5 & 1 & 8 & 4 & D116E \\
\hline 59145 & 64 & 16 & 1 & 1 & 0.25 & 4 & 2 & D116E \\
\hline 57464 & 64 & 16 & 1 & 0.25 & 0.25 & 2 & 4 & D116E E266D \\
\hline 59537 & 64 & 0.0625 & 0.125 & 0.625 & 0.125 & 4 & 0.5 & D116E E266D V488I \\
\hline 59407 & 64 & 1 & 0.25 & 0.25 & 0.25 & 4 & 2 & D116E V488I \\
\hline 57856 & 64 & 0.125 & 1 & 0.25 & 0.125 & 4 & 4 & D11DE E266D V488I \\
\hline 56682 & 64 & 16 & 16 & 1 & 0.125 & 4 & 1 & D225H E266D \\
\hline 56517 & 64 & 16 & 16 & 2 & 0.125 & 4 & 2 & E266D \\
\hline 56689 & 64 & 0.0313 & 0.125 & 0.625 & 0.125 & 4 & 2 & E266D \\
\hline 56262 & 64 & 1 & 16 & 1 & 8 & 4 & 4 & E266D V437I V488I \\
\hline 59679 & 64 & 16 & 0.5 & 2 & 0.25 & 4 & 2 & E266D V488I \\
\hline 56214 & 64 & 4 & 16 & 0.5 & 0.25 & 8 & 4 & E266D V488I \\
\hline 57442 & 64 & 0.125 & 0.5 & 1 & 0.25 & 4 & 0.5 & K342R \\
\hline 55475 & 64 & 64 & 0.25 & 0.25 & 0.125 & 0.125 & 4 & V437I \\
\hline
\end{tabular}

Abbreviations: B, amphotericin B; CLOT, clotrimazole; FLZ, fluconazole; ITZ, itraconazole; KETO, ketoconazole; NYS, nystain.

${ }^{a}$ Isolates with no missense mutation in ERG11.

Azoles inhibit the enzyme lanosterol $14 \alpha$-demethylase, which is encoded by the ERG11 gene. A mutation in the ERG11 gene or its overexpression may affect the enzyme's affinity for drugs, resulting in resistance (10). However, in our previous study, 63.27\% (31/49) of the fluconazoleresistant isolates were not associated with point mutations in the ERG11 gene, indicating that other factors are involved in azole resistance in these strains. In this study, we further investigated the relationship between ERG11 expression/mutation and fluconazole resistance. The overexpres- sion of the ERG11 gene may increase the production of drug target enzymes to an extent exceeding the inhibitory capacity of antifungal drugs, which may, in turn, contribute to fluconazole resistance. However, the role of overexpression of ERG11 in fluconazole resistance remains enigmatic. Some studies have suggested that the overexpression of ERG11 is not significantly related to fluconazole resistance in C. albicans (33). Similar to previous reports, we did not find any significant difference in the expression of ERG11 between multi-azole susceptible and resistant strains in this 

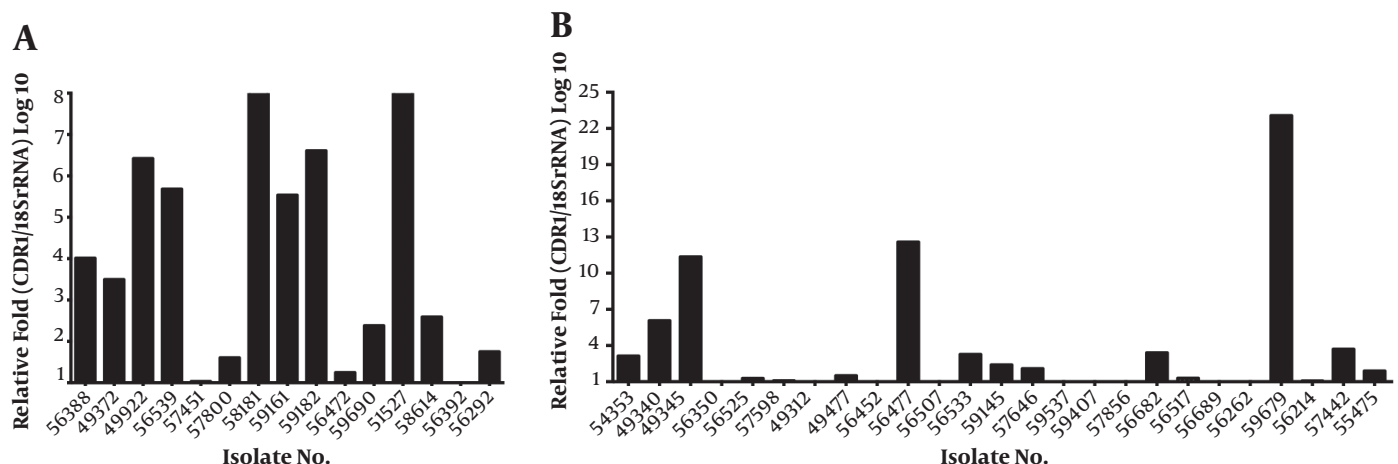

Figure 1. The CDR1 gene upregulation in fluconazole-resistant isolates. A, CDR1 gene expression in fluconazole-resistant isolates that were associated with ERG11 gene point mutations; $B, C D R 1$ gene expression in fluconazole-resistant isolates that were not associated with ERG11 gene point mutations.
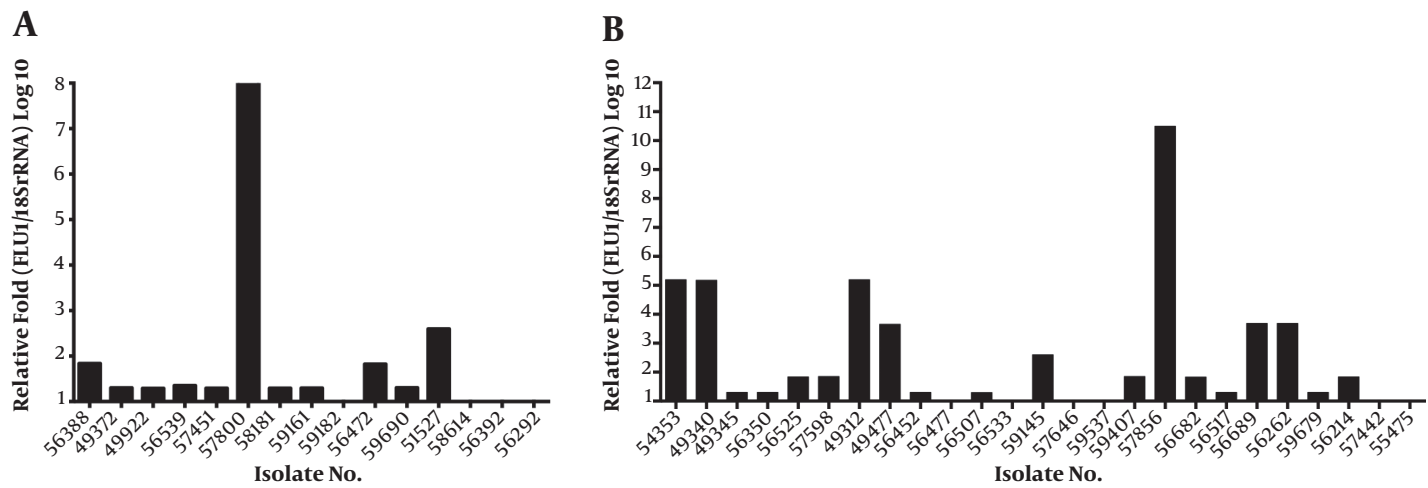

Figure 2. The FLU1 gene upregulation in fluconazole-resistant isolates. A, FLU1 gene expression in fluconazole-resistant isolates that were associated with ERG11 gene poin mutations; B, FLU1 gene expression in fluconazole-resistant isolates that were not associated with ERG11 gene point mutations.
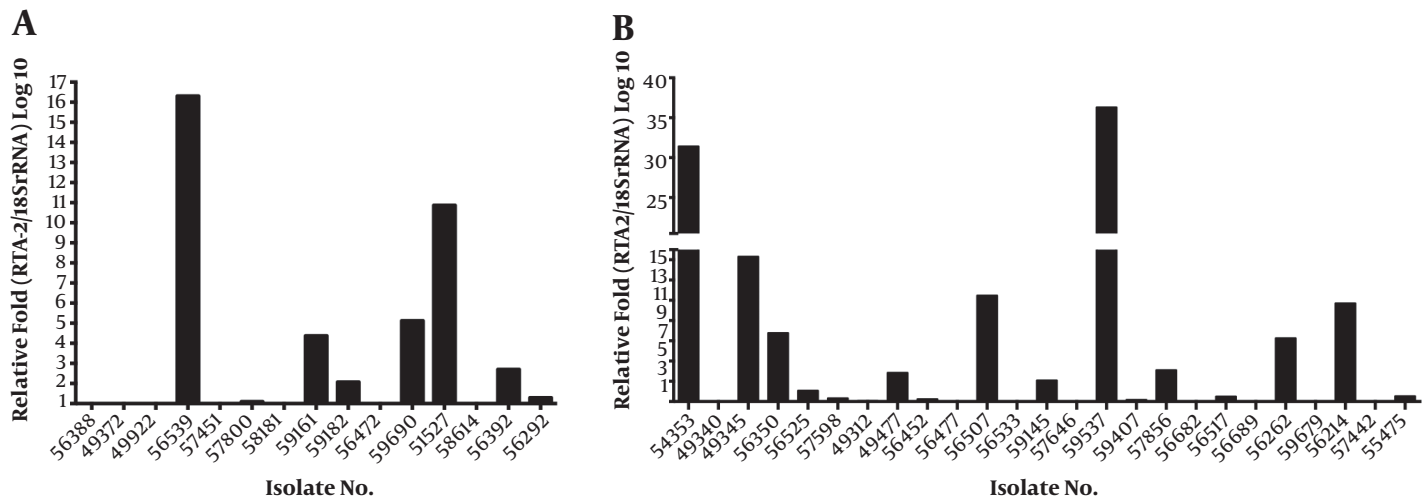

Figure 3. RTA2 gene overexpression in fluconazole-resistant isolates. A, RTA2 gene expression in fluconazole-resistant isolates that were associated with ERG11 gene point mutations; B, CDR1 gene expression in fluconazole-resistant isolates that were not associated with ERG11 gene point mutations.

study.

In Candida, another important mechanism of drug resistance is associated with efflux transporters. In this study, we aimed to elucidate the role of efflux transporters in the development of fluconazole resistance. As previously reported, the overexpression of the CDR gene is one of the 
most predominant mechanisms of MDR in azole-resistant Candida clinical isolates. Prior studies found that a drug efflux pump-encoding $C D R$ gene contributed to the development of the cross-resistance phenotype in C. glabrata strains (34). Mdr1p, encoded by the MDR gene, is a member of the MFS transporters, and is able to pump several structurally unrelated compounds out of the cell, including fluconazole. The expression levels of CDR1, CDR2, and MDR1 genes increased in most clinical isolates with fluconazole MICs of $\geq 64 \mu \mathrm{g} / \mathrm{mL}$, while the disruption of these genes resulted in hypersensitivity to azoles (34). Furthermore, the increased expression of CDR1, CDR2, and MDR1 genes was a major contributor to azoles resistance in clinical isolates $(21,35-38)$. In this study, our results suggested that the CDR1 gene was upregulated in fluconazole-resistant isolates. However, unlike most reports, no correlation was observed between MDR and overexpression of CDR2 and MDR1 in this study.

FLU1, encoding Flu1p, is a multidrug efflux transporter implicated in mycophenolic acid resistance. Similar to $C D R, F L U 1$ was discovered in a genomic library screened for the complementation of fluconazole hyper-susceptibility in the Pdr5 (ABC transporter gene) mutant Saccharomyces cerevisiae isolates (20). When the FLU1 gene was heterologously expressed in S. cerevisiae, it mediated both fluconazole and cycloheximide resistance (20). The deletion of FLU1 leads to insignificant changes in susceptibility to fluconazole. However, the deletion of FLU1 in a strain based on the disruption of other genes encoding multidrug efflux pumps (such as CDR1, CDR2, and MDR) may cause increased susceptibility to several azole derivatives (20). Similarly, the gene product of FLU1 has been found to mimic Tpo1 from $S$. cerevisiae, which is a primary plasma membrane polyamine efflux transporter (39). Further study found that FLU1 is able to pump Histatin 5 out of the cell and reduce the toxicity of Histatin 5 in C. albicans (40). However, the overexpression of FLU1 is generally uncommon among clinical resistant isolates of $C$. albicans. In most studies, changes in the expression level of FLU1 were not significant between azole-resistant and susceptible C. albicans isolates $(21,37)$. Interestingly, our findings showed that upregulation of FLU1 was one of the dominant mechanisms in the fluconazole-resistant isolates analyzed in this study.

Several lines of evidence suggest that fluconazole resistance may involve many unknown mechanisms that have yet to be elucidated. Recently, studies found that calcium signaling plays an important role in the development of drug resistance and it may be a target for overcoming drug resistance $(41,42)$. A novel gene, RTA2, which mediates calcineurin-dependent resistance to azoles, was found to contribute to the development of fluconazole resistance $(23,43)$. The knockdown of RTA2 leads to higher suscepti- bility of C. albicans to fluconazole. Conversely, ectopic expression of RTA2 resulted in markedly decreased fluconazole efficacy in mice with systemic Candida infections (44). Furthermore, previous studies found that the RTA2 gene was over-expressed in both laboratory and clinical resistant strains (45). Consistently, the RTA2 expression levels elevated in our present study.

Our previous results showed that only 36.73\% of fluconazole-resistant strains were associated with point mutations in ERG11. Interestingly, we also found that $75.0 \%$ of the fluconazole-resistant isolates exhibited the overexpression of FLU1 gene, 62.5\% were associated with upregulation of CDR1 (more than 1.5-fold expression), and $45 \%$ showed high levels of RTA2 expression. In addition, we found that $10 \%$ of the fluconazole-resistant isolates were associated simultaneously with ERG11 mutation and overexpression of RTA2, CDR1, and FLU1 genes.

These results indicate that multiple genes are associated with fluconazole resistance. Additionally, the upregulation of CDR1 was a major mechanism in fluconazoleresistant isolates having point mutations in ERG11. Interestingly, we found that more than $90 \%$ of the fluconazoleresistant isolates with A114S and Y132H ERG11 variants exhibited the upregulated expression of CDR1. It seems that the specific ERG11 mutant variants A114S and Y132H may be associated with CDR1 overexpression. However, further studies need to investigate the relationship between CDR1 expression and ERG11 variants A114S and Y132H. For the isolates that were not related to mutant variants of $E R G 11$, the overexpression of FLU1 and RTA2 was a major contributor to drug resistance.

\subsection{Conclusions}

In conclusion, we demonstrated that the overexpression of FLU1 and RTA2 was correlated with azole resistance; this finding had not been reported previously in clinical isolates of C. albicans. Taken together, this study provides useful information for the treatment of candidiasis and indicates that clinicians should be cautious of crossresistance within this class of antifungal drugs, especially for the treatment of patients with prior azoles prophylaxis or patients at high risk of C. albicans infections. Moreover, fluconazole resistance in C. albicans is a multifactorial phenomenon with complicated mechanisms. Therefore, it is important to notice that most of these mechanisms are frequently combined in a single isolate to contribute to a stepby-step acquisition of fluconazole resistance.

\section{Acknowledgments}

We thank MD Xuefei Hu and the staff of the Department of Clinical Laboratory of the First Affiliated Hospital of Nan- 
chang University for the supply of clinical isolates.

\section{Footnotes}

Authors' Contribution: Hongxia Zhang and Qiufang Xu contributed equally to this study. Study concept and design: Shibo Huang and Xiao-Tian Huang. Acquisition of data: Hongxia Zhang and Qiufang Xu. Analysis and interpretation of data: Hongxia Zhang, Qiufang Xu, and Ying Ying. Drafting of the manuscript: Shibo Huang. Critical revision of the manuscript for important intellectual content: Xiao-Tian Huang and Ying Ying. Statistical analysis: Suchen Li and Zhiqin Zhang. Administrative, technical, and material support: Lingbing Zeng. Study supervision: Shibo Huang and Xiao-Tian Huang.

Conflict of Interests: All authors report no conflicts of interest relevant to this paper.

Ethical Approval: The study was approved by the Ethics Committee of the First Affiliated Hospital of Nanchang University (Nanchang, China [approval number: 2014036]).

Financial Disclosure: It is not declared by the authors.

Funding/Support: This study was supported by the National Natural Science Foundation of China (No. 31660035 and No.81560299), the Science and Technology Foundation of Jiangxi Province (20171ACB20003 and 20192ACBL21042), and the Innovation Fund Designated for Graduate Students of Nanchang University (CX2019164).

\section{References}

1. Sydnor ER, Perl TM. Hospital epidemiology and infection control in acute-care settings. Clin Microbiol Rev. 2011;24(1):141-73. doi: 10.1128/CMR.00027-10. [PubMed: 21233510]. [PubMed Central: PMC3021207].

2. Alizadeh M, Kolecka A, Boekhout T, Zarrinfar H, Ghanbari Nahzag MA, Badiee $\mathrm{P}$, et al. Identification of Candida species isolated from vulvovaginitis using matrix assisted laser desorption ionizationtime of flight mass spectrometry. Curr Med Mycol. 2017;3(4):21-5. doi: 10.29252/cmm.3.4.21. [PubMed: 29707675]. [PubMed Central: PMC5917097].

3. Zarrinfar H, Kaboli S, Dolatabadi S, Mohammadi R. Rapid detection of Candida species in bronchoalveolar lavage fluid from patients with pulmonary symptoms. Braz J Microbiol. 2016;47(1):172-6. doi: 10.1016/j.bjm.2015.02.001. [PubMed: 26887241]. [PubMed Central: PMC4822774].

4. Esmailzadeh A, Zarrinfar H, Fata A, Sen T. High prevalence of candiduria due to non-albicans Candida species among diabetic patients: A matter of concern? J Clin Lab Anal. 2018;32(4). e22343. doi: 10.1002/jcla.22343. [PubMed: 29076587].

5. Cowen LE. The evolution of fungal drug resistance: Modulating the trajectory from genotype to phenotype. Nat Rev Microbiol. 2008;6(3):187-98. doi: 10.1038/nrmicro1835. [PubMed: 18246082].

6. Anderson JB. Evolution of antifungal-drug resistance: Mechanisms and pathogen fitness. Nat Rev Microbiol. 2005;3(7):547-56. doi: 10.1038/nrmicro1179. [PubMed: 15953931].
7. Sanguinetti M, Posteraro B, Lass-Florl C. Antifungal drug resistance among Candida species: Mechanisms and clinical impact. Mycoses. 2015;58 Suppl 2:2-13. doi: 10.111/myc.12330. [PubMed: 26033251].

8. Berkow EL, Lockhart SR. Fluconazole resistance in Candida species: A current perspective. Infect Drug Resist. 2017;10:237-45. doi: 10.2147/IDR.S118892. [PubMed: 28814889]. [PubMed Central: PMC5546770].

9. Sardari A, Zarrinfar H, Mohammadi R. Detection of ERG11 point mutations in Iranian fluconazole-resistant Candida albicans isolates. Curr Med Mycol. 2019;5(1):7-14. doi: 10.18502/cmm.5.1.531. [PubMed: 31049452]. [PubMed Central: PMC6488286].

10. Feng W, Yang J, Xi Z, Qiao Z, Lv Y, Wang Y, et al. Mutations and/or overexpressions of ERG4 and ERG11 genes in clinical azoles-resistant isolates of Candida albicans. Microb Drug Resist. 2017;23(5):563-70. doi: 10.1089/mdr.2016.0095. [PubMed: 27976986].

11. Li QQ, Tsai HF, Mandal A, Walker BA, Noble JA, Fukuda Y, et al. Sterol uptake and sterol biosynthesis act coordinately to mediate antifungal resistance in Candida glabrata under azole and hypoxic stress. Mol Med Rep. 2018;17(5):6585-97. doi: 10.3892/mmr.2018.8716. [PubMed: 29532896]. [PubMed Central: PMC5928633].

12. Prasad R, Rawal MK, Shah AH. Candida efflux ATPases and antiporters in clinical drug resistance. Adv Exp Med Biol. 2016;892:351-76. doi 10.1007/978-3-319-25304-6_15. [PubMed: 26721282].

13. K. Redhu A, Shah AH, Prasad R. MFS transporters of Candida species and their role in clinical drug resistance. FEMS Yeast Res. 2016;16(4). doi: 10.1093/femsyr/fow043. [PubMed: 27188885].

14. Prasad R, Banerjee A, Khandelwal NK, Dhamgaye S. The ABCs of Candida albicans multidrug transporter Cdr1. Eukaryot Cell. 2015;14(12):1154-64. doi: 10.1128/EC.00137-15. [PubMed: 26407965]. [PubMed Central: PMC4664872].

15. Franz R, Kelly SL, Lamb DC, Kelly DE, Ruhnke M, Morschhauser J. Multiple molecular mechanisms contribute to a stepwise development of fluconazole resistance in clinical Candida albicans strains. Antimicrob Agents Chemother. 1998;42(12):3065-72. [PubMed: 9835492]. [PubMed Central: PMC106000].

16. Krishnamurthy S, Gupta V, Prasad R, Panwar SL, Prasad R. Expression of CDR1, a multidrug resistance gene of Candida albicans: transcriptional activation by heat shock, drugs and human steroid hormones. FEMS Microbiol Lett. 1998;160(2):191-7. doi: 10.1111/j.15746968.1998.tb12910.x. [PubMed: 9532737].

17. Marr KA, Lyons CN, Rustad TR, Bowden RA, White TC. Rapid, transient fluconazole resistance in Candida albicans is associated with increased mRNA levels of CDR. Antimicrob Agents Chemother. 1998;42(10):2584-9. [PubMed: 9756759]. [PubMed Central: PMC105901].

18. Sanglard D, Ischer F, Monod M, Bille J. Susceptibilities of Candida albicans multidrug transporter mutants to various antifungal agents and other metabolic inhibitors. Antimicrob Agents Chemother. 1996;40(10):2300-5. [PubMed: 8891134]. [PubMed Central: PMC163524].

19. Sanglard D, Ischer F, Monod M, Bille J. Cloning of Candida albicans genes conferring resistance to azole antifungal agents: characterization of CDR2, a new multidrug ABC transporter gene. Microbiology. 1997;143 ( Pt 2):405-16. doi: 10.1099/00221287-143-2-405. [PubMed: 9043118].

20. Calabrese D, Bille J, Sanglard D. A novel multidrug efflux transporter gene of the major facilitator superfamily from Candida albicans (FLU1) conferring resistance to fluconazole. Microbiology. 2000;146 ( Pt 11):2743-54. doi: 10.1099/00221287-146-11-2743. [PubMed: 11065353].

21. Pourakbari B, Teymuri M, Mahmoudi S, Valian SK, Movahedi Z, Eshaghi $\mathrm{H}$, et al. Expression of major efflux pumps in fluconazoleresistant Candida albicans. Infect Disord Drug Targets. 2017;17(3):178-84. doi: 10.2174/1871526517666170531114335. [PubMed: 28558643].

22. Morio F, Jensen RH, Le Pape P, Arendrup MC. Molecular basis of antifungal drug resistance in yeasts. Int $J$ Antimicrob Agents. 
2017;50(5):599-606. doi: 10.1016/j.ijantimicag.2017.05.012. [PubMed: 28669835].

23. Jia XM, Ma ZP, Jia Y, Gao PH, Zhang JD, Wang Y, et al. RTA2, a novel gene involved in azole resistance in Candida albicans. Biochem Biophys Res Commun. 2008;373(4):631-6. doi: 10.1016/j.bbrc.2008.06.093. [PubMed: 18601908].

24. Ying Y, Zhao Y, Hu X, Cai Z, Liu X, Jin G, et al. In vitro fluconazole susceptibility of 1,903 clinical isolates of Candida albicans and the identification of ERG11 mutations. Microb Drug Resist. 2013;19(4):266-73. doi: 10.1089/mdr.2012.0204. [PubMed: 23484590].

25. Clinical and Laboratory Standards Institute (CLSI). Reference method for broth dilution antifungal susceptibility testing of yeasts. 3rd ed. Wayne: Clinical and Laboratory Standards Institute; 2008.

26. Martel CM, Parker JE, Bader O, Weig M, Gross U, Warrilow AG, et al. A clinical isolate of Candida albicans with mutations in ERG11 (encoding sterol 14alpha-demethylase) and ERG5 (encoding C22 desaturase) is cross resistant to azoles and amphotericin B. Antimicrob Agents Chemother. 2010;54(9):3578-83. doi: 10.1128/AAC.00303-10. [PubMed: 20547793]. [PubMed Central: PMC2934972].

27. Davis MJ, Goldberg JB. Purification and visualization of lipopolysaccharide from Gram-negative bacteria by hot aqueous-phenol extraction. J Vis Exp. 2012;(63). doi: 10.3791/3916. [PubMed: 22688346] [PubMed Central: PMC3466933].

28. Bineshian F, Yadegari MH, Sharifi Z, Akbari Eidgahi M, Nasr R. Identification of Candida species using MP65 gene and evaluation of the Candida albicans MP65 gene expression in BALB/C Mice. Jundishapur J Microbiol. 2015;8(5). e18984. doi: 10.5812/jjm.8(5)2015.18984. [PubMed: 26060567]. [PubMed Central: PMC4458353].

29. Wang Y, Yang Q, Chen L, Liu L, Hao R, Zhang T, et al. Crossresistance between voriconazole and fluconazole for non-albicans Candida infection: A case-case-control study. Eur J Clin Microbiol Infect Dis. 2017;36(11):2117-26. doi: 10.1007/s10096-017-3034-4. [PubMed: 28620845].

30. Warnock DW. Azole drug resistance in Candida species. J Med Microbiol. 1992;37(4):225-6. doi: 10.1099/00222615-37-4-225. [PubMed 1404317].

31. Mane A, Vidhate P, Kusro C, Waman V, Saxena V, Kulkarni-Kale U, et al. Molecular mechanisms associated with Fluconazole resistance in clinical Candida albicans isolates from India. Mycoses. 2016;59(2):93100. doi: 10.1111/myc.12439. [PubMed: 26648048]

32. Roy RL. Drug resistance in tuberculosis. J Indian Med Assoc. 1970;55(10):365-6. [PubMed: 5501196].

33. Park S, Perlin DS. Establishing surrogate markers for fluconazole resistance in Candida albicans. Microb Drug Resist. 2005;11(3):232-8. doi 10.1089/mdr.2005.11.232. [PubMed:16201925].

34. Chakrabarti A, Chatterjee SS, Rao KL, Zameer MM, Shivaprakash MR, Singhi S, et al. Recent experience with fungaemia: Change in species distribution and azole resistance. Scand JInfect Dis. 2009;41(4):275-84. doi:10.1080/00365540902777105. [PubMed: 19229762].

35. You L, Qian W, Yang Q, Mao L, Zhu L, Huang X, et al. ERG11 gene mutations and MDR1 upregulation confer pan-azole resistance in Candida tropicalis causing disseminated Candidiasis in an acute lymphoblastic leukemia patient on posaconazole prophylaxis. Antimicrob Agents Chemother. 2017;61(7). doi: 10.1128/AAC.02496-16. [PubMed: 28507109]. [PubMed Central: PMC5487663].

36. Holmes AR, Lin $\mathrm{YH}$, Niimi K, Lamping E, Keniya M, Niimi M, et al. ABC transporter Cdr1p contributes more than Cdr2p does to fluconazole efflux in fluconazole-resistant Candida albicans clinical isolates. Antimicrob Agents Chemother. 2008;52(11):3851-62. doi: 10.1128/AAC.00463-08. [PubMed: 18710914]. [PubMed Central: PMC2573144].

37. Tsao S, Rahkhoodaee F, Raymond M. Relative contributions of the Candida albicans $\mathrm{ABC}$ transporters Cdr1p and Cdr2p to clinical azole resistance. Antimicrob Agents Chemother. 2009;53(4):1344-52. doi: 10.1128/AAC.00926-08. [PubMed: 19223631]. [PubMed Central: PMC2663127].

38. Shahrokhi S, Noorbakhsh F, Rezaie S. Quantification of CDR1 gene expression in fluconazole resistant Candida glabrata strains using realtime PCR. Iran J Public Health. 2017;46(8):1118-22. [PubMed: 28894714]. [PubMed Central: PMC5575392].

39. Braun BR, van Het Hoog M, d'Enfert C, Martchenko M, Dungan J, Kuo A, et al. A human-curated annotation of the Candida albicans genome. PLoS Genet. 2005;1(1):36-57. doi: 10.1371/journal.pgen.0010001. [PubMed: 16103911]. [PubMed Central: PMC1183520].

40. Li R, Kumar R, Tati S, Puri S, Edgerton M. Candida albicans flu1mediated efflux of salivary histatin 5 reduces its cytosolic concentration and fungicidal activity. Antimicrob Agents Chemother. 2013;57(4):1832-9. doi: 10.1128/AAC.02295-12. [PubMed: 23380720]. [PubMed Central: PMC3623299].

41. Li X, Sun S. Targeting the fungal calcium-calcineurin signaling network in overcoming drug resistance. Future Med Chem. 2016;8(12):1379-81. doi: 10.4155/fmc-2016-0094. [PubMed: 27463738].

42. Luna-Tapia A, Tournu H, Peters TL, Palmer GE. Endosomal trafficking defects can induce calcium-dependent azole tolerance in Candida albicans. Antimicrob Agents Chemother. 2016;60(12):7170-7. doi: 10.1128/AAC.01034-16. [PubMed: 27645241]. [PubMed Central: PMC5118996].

43. Thomas E, Sircaik S, Roman E, Brunel JM, Johri AK, Pla J, et al. The activity of RTA2, a downstream effector of the calcineurin pathway, is required during tunicamycin-induced ER stress response in Candida albicans. FEMS Yeast Res. 2015;15(8). doi: 10.1093/femsyr/fov095. [PubMed: 26518191].

44. Jia Y, Tang RJ, Wang L, Zhang X, Wang Y, Jia XM, et al. Calciumactivated-calcineurin reduces the in vitro and in vivo sensitivity of fluconazole to Candida albicans via Rta2p. PLoS One. 2012;7(10). e48369. doi: 10.1371/journal.pone.0048369. [PubMed: 23118995]. [PubMed Central: PMC3484117].

45. Xu Z, Zhang LX, Zhang JD, Cao YB, Yu YY, Wang DJ, et al. cDNA microarray analysis of differential gene expression and regulation in clinically drug-resistant isolates of Candida albicans from bone marrow transplanted patients. Int J Med Microbiol. 2006;296(6):421-34. doi: 10.1016/j.ijmm.2006.03.004. [PubMed:16782404]. 